\title{
On Nodal Sets for Dirac and Laplace Operators
}

\author{
Christian Bär *
}

March, 1997

\begin{abstract}
We prove that the nodal set (zero set) of a solution of a generalized Dirac equation on a Riemannian manifold has codimension 2 at least. If the underlying manifold is a surface, then the nodal set is discrete. We obtain a quick proof of the fact that the nodal set of an eigenfunction for the Laplace-Beltrami operator on a Riemannian manifold consists of a smooth hypersurface and a singular set of lower dimension. We also see that the nodal set of a $\Delta$-harmonic differential form on a closed manifold has codimension 2 at least; a fact which is not true if the manifold is not closed. Examples show that all bounds are optimal.
\end{abstract}

Mathematics Subject Classification: 58G03, 35B05

Keywords: generalized Dirac operator, Dirac equation, Laplace operator, nodal set, harmonic differential form

\section{Introduction}

The motion of a vibrating membrane $M$ fixed at the boundary is described by a function $u: M \times \mathbb{R} \rightarrow \mathbb{R}$ satisfying the wave equation

$$
\frac{\partial^{2} u}{\partial t^{2}}+\Delta u=0
$$

and Dirichlet boundary conditions $\left.u\right|_{\partial M}=0$. One can expand $u$ into a series $u(x, t)=\sum_{j=0}^{\infty}\left(a_{j} \sin \left(\sqrt{\lambda_{j}} t\right)+b_{j} \cos \left(\sqrt{\lambda_{j}} t\right)\right) \phi_{j}(x)$ where $\phi_{j}$ are the $\Delta$ eigenfunctions on $M$ for the eigenvalue $\lambda_{j}$. A "pure sound" is given by $u(x, t)=$ $\left(a_{j} \sin \left(\sqrt{\lambda_{j}} t\right)+b_{j} \cos \left(\sqrt{\lambda_{j}} t\right)\right) \phi_{j}(x)$ for some fixed $j$. The zero set of $\phi_{j}$ describes those points of the membrane which do not move during the vibration. They can be made visible by putting fine powder on the membrane.

The structure of the zero set of eigenfunctions of the Laplace-Beltrami operator on surfaces is well understood. They consist of smooth arcs, called nodal

*Partially supported by SFB 256 and by the GADGET program of the EU 
lines, and isolated singular points where these arcs meet. One knows that the arcs meeting at a singular point form an equiangular configuration, see [1] or [7, Satz 1] for a proof. One also has lower and upper bounds for the length of the nodal lines [0], Satz 2], [17, Theorem 1.2, Corollary 1.3], [13, Theorem 4.2].

Such a precise understanding of nodal sets seems difficult in higher dimensions. But one has the following regularity result.

If $\phi$ is an eigenfunction of the Laplace-Beltrami operator on an $n$-dimensional Riemannian manifold, then the nodal set of $\phi$ consists of a smooth hypersurface and a singular part of dimension $\leq n-2$.

Essentially this fact has been stated as Theorem 2.2 in [12]. Once it is established the standard proof of Courant's nodal domain theorem in dimension 2 carries over to higher dimensions. A nodal domain is a connected component of the complement of the nodal set. Courant's nodal domain theorem states that the number of nodal domains of the $i^{t h}$ eigenfunction of $\Delta$ is less than or equal to $i$. The point is that one has to use a Green formula over a nodal domain and this requires some regularity of its boundary.

It was pointed out by Y. Colin de Verdière that the proof of Theorem 2.2 in [12] has a serious gap, see [3, App. E]. Therefore Bérard and Meyer modified the proof of Courant's nodal domain theorem. They approximate the nodal domains by regular domains [3, App. D].

The above regularity statement on Laplace-eigenfunctions has (to our knowledge) first been proved by R. Hardt and L. Simon in [18, Theorem 1.10], see also [8] for a special case. We will obtain a quick proof of this fact once our theorem on the nodal set of solutions of Dirac equations is established (Corollary 2).

One has estimates for the $(n-1)$-dimensional Hausdorff measure of the nodal set, see [14, Theorem 4.2], [15, Theorem 1.2], [16], and [18, Theorem 5.3], some of which only work if the Riemannian metric is assumed to be real analytic. There are also estimates for the volume of the nodal domains, see [21, Theorem B], [10, Theorem 2].

Not much is known about the topology of the singular set. The problem is that in dimension $n \geq 3$ the nodal set need not be locally homeomorphic to its tangent cone. This precisely was the problem in [12]. For a structural result in dimension $n=3$ see [11, Theorem 1.2].

The purpose of this paper is to study the nodal set of solutions of certain systems of elliptic linear partial differential equations of first order, generalized Dirac equations. Precise definitions will be given in the next section.

To get some feeling of what to expect let us first look at the trivial onedimensional case. A Laplace equation is then nothing but a second order linear ordinary differential equation. The standard theory tells us that zeros of solutions must be isolated. A Dirac equation becomes a first order linear ordinary differential equation and we see that nontrivial solutions have no zeros at all. 
Another interesting test case is provided by holomorphic functions on Riemann surfaces. The Cauchy-Riemann equations are special generalized Dirac equations. As is well-known, zeros of solutions must form a discrete set. In higher dimensions, a holomorphic function defines a complex subvariety having real codimension 2 .

This, as well as other special cases, leads us towards the conjecture that the nodal set of a solution of a generalized Dirac equation on an $n$-dimensional manifold has dimension $\leq n-2$.

The main result of this paper says that this conjecture is indeed true. Examples show that this bound is optimal. In the two-dimensional case we have a similar unique-continuation theorem as we have for holomorphic functions (Corollary 3). If the nodal set of a solution of a generalized Dirac equation on a connected surface has a cumulation point, then this solution must vanish identically.

There is a physical interpretation in quantum mechanics similar to the vibrating membrane for the Laplace equation. The "wave function" of a fermion (e.g. an electron) is given by a spinor field, in the simplest case by a function $\psi: \mathbb{R}^{3} \times \mathbb{R} \rightarrow \mathbb{C}^{2}$, satisfying the equation

$$
i \frac{\partial}{\partial t} \psi+(D+h) \psi=0
$$

where $D$ is the Dirac operator on $\mathbb{R}^{3}$ and $h$ is a potential. The particle is in "pure state" if $\psi$ has the form $\psi(x, t)=e^{i \lambda t} \Psi(x)$ where $\Psi$ is an eigenfunction of $D+h$ for the eigenvalue $\lambda$. The scalar function $|\psi(x, t)|^{2}=|\Psi(x)|^{2}$ can be interpreted as the probability measure for the particle to be found at the point $x$. Hence the nodal set of $\Psi$ is the set of points where this probability measure is zero. Our main result then says that this "exclusive set" is not very big, it is at most one-dimensional.

We believe that the nodal set of solutions of Dirac equations very often carries important information about the underlying manifold. In the special case of holomorphic functions on complex manifolds this is classical. Recently, Taubes has given an impressive example in the theory of 4-dimensional symplectic manifolds. Starting from solutions $\psi_{r}$ of a one-parameter family of Dirac equations (deformed first Seiberg-Witten equation), $r \in \mathbb{R}$, he constructs pseudoholomorphic curves. Philosophically, these curves are given by the zero locus of $\psi_{\infty}$ (which is 2-dimensional!). See [23], [24], and [19] for a very readable survey.

It is nice that information about solutions of Dirac equations yields also information about solutions of Laplace equations. It was mentioned earlier that we will obtain a quick proof of the fact that the nodal set of a $\Delta$-eigenfunction is the union of a smooth hypersurface and a singular part of dimension $\leq n-2$.

We will also see that the nodal set of a $\Delta$-harmonic differential form on a closed manifold has codimension 2 at least (Corollary 1). This is surprising since it is not true if the underlying manifold is not closed nor does it hold for other $\Delta$-eigenforms even if the manifold is closed. 
The paper is organized as follows. In the next section we give precise definitions and we collect a few well-known facts about Dirac operators for later use. We then formulate the main result.

In the third section we give the most important examples for generalized Dirac operators and for some of these we draw conclusions from our main theorem.

In section 4 we give the proof. We employ tools similar to those used in complex algebraic geometry when one studies the topological structure of complex algebraic varieties. We use an analog of Weierstrass' preparation theorem for differentiable functions due to Malgrange to write a solution of a Dirac equation in a certain normal form. This theorem is important e.g. in catastrophe theory. To insure that we can apply this theorem we have to use Aronszajn's unique continuation theorem. We easily conclude that the nodal set has dimension not bigger than $n-1$.

The difficult part is to show that simultaneous vanishing of several components of our section must reduce the dimension of its zero set once more. This requires a careful investigation of certain resultants and this is where we really use the Dirac equation.

\section{Statement of Result}

Let $M$ be an $n$-dimensional Riemannian manifold, let $S$ be a Riemannian or Hermitian vector bundle over $M$ on which the Clifford bundle $C l(T M)$ acts from the left. This means that at every point $p \in M$ there is a linear map $T_{p} M \otimes S_{p} \rightarrow$ $S_{p}, v \otimes s \rightarrow v \cdot s$, satisfying the relations

$$
v \cdot w \cdot s+w \cdot v \cdot s=-2\langle v, w\rangle s
$$

and

$$
\left\langle v \cdot s_{1}, s_{2}\right\rangle=-\left\langle s_{1}, v \cdot s_{2}\right\rangle .
$$

Moreover, let $\nabla$ be a metric connection on $S$ satisfying the Leibniz rule for Clifford multiplication

$$
\nabla(v \cdot s)=(\nabla v) \cdot s+v \cdot \nabla s .
$$

Such an $S$ is called a Dirac bundle. All geometric data such as metrics and Clifford multiplication are assumed to be $C^{\infty}$-smooth.

The (generalized) Dirac operator acts on sections of $S$ and is defined by

$$
D s=\sum_{i=1}^{n} e_{i} \cdot \nabla_{e_{i}} s
$$

where $e_{1}, \ldots, e_{n}$ denote a local orthonormal frame of $T M$. The Dirac operator is easily seen to be independent of the choice of orthonormal frame. It is a first order formally self-adjoint elliptic differential operator, see [4] or [20] for details. 
Three general facts about Dirac operators will be used later on and should be stated here for completeness. The proofs are simple computations.

1. If $s$ is a differentiable section of $S$ and $f$ a differentiable function on $M$, then the following Leibniz rule holds [20, p. 116, Lemma 5.5]:

$$
D(f \cdot s)=f \cdot D s+\nabla f \cdot s
$$

where $\nabla f$ is the gradient of $f$.

2. The connection $\nabla$ maps sections of $S$ into those of $T^{*} M \otimes S$. Denote the formal adjoint of this operator by $\nabla^{*}$. Then we have the following Weitzenböck formula [20, p. 155, Theorem 8.2]:

$$
D^{2}=\nabla^{*} \nabla+\Re
$$

where $\Re$ is an endomorphism field given by curvature. Operators of the form $\nabla^{*} \nabla+$ endomorphism field are called generalized Laplacians.

3. If $M$ has smooth boundary $\partial M$ with outer unit normal field $\nu$, if $s_{1}$ and $s_{2}$ are compactly supported $C^{1}$-sections of $S$, then the following Green formula holds [20, p. 115, eq. (5.7)]:

$$
\left(D s_{1}, s_{2}\right)_{L^{2}(M)}-\left(s_{1}, D s_{2}\right)_{L^{2}(M)}=\int_{\partial M}\left\langle\nu \cdot s_{1}, s_{2}\right\rangle .
$$

If $s$ is an eigensection of $D$, i.e. $D s=\lambda s, \lambda \in \mathbb{R}$, or, more generally, $s$ satisfies $(D+h) s=0$ for some endomorphism field $h$ of $S$, then the zero locus of $s$, $\{x \in M \mid s(x)=0\}$, is called the nodal set of $s$. The main purpose of this paper is to study the structure of such nodal sets.

Main Theorem. Let $M$ be a connected $n$-dimensional Riemannian manifold with Dirac bundle $S$ and generalized Dirac operator D. Let $h$ be a smooth endomorphism field for $S$ and let $s \not \equiv 0$ be a solution of

$$
(D+h) s=0 \text {. }
$$

Then the nodal set of $s$ is a countably $(n-2)$-rectifiable set and thus has Hausdorff dimension $n-2$ at most.

If $n=2$, then the nodal set of $s$ is a discrete subset of $M$.

Recall that a subset of an $n$-dimensional Riemannian manifold $M$ is called countably $k$-rectifiable if it can be written as a countable union of sets of the form $\Phi(X)$ where $X \subset \mathbb{R}^{k}$ is bounded and $\Phi: X \rightarrow M$ is a Lipschitz map. The proof will be given in the fourth section. 


\section{Examples and Consequences}

Let us look at the most important examples and draw some conclusions.

Example 1. The Clifford algebra bundle $C l(T M)$ acts on itself by Clifford multiplication. As a vector bundle $C l(T M)$ can be canonically identified with the exterior form bundle $\Lambda^{*}(T M)$. We thus obtain a real Dirac bundle $S=\Lambda^{*}(T M)$ with Levi-Civita connection $\nabla$. The Dirac operator is $D=d+\delta$ where $d$ denotes exterior differentiation and $\delta$ codifferentiation.

Example 2. Let $M$ be a spin manifold. Then there exists the spinor bundle $S$, a complex Dirac bundle of rank $2^{[n / 2]}$. The connection $\nabla$ is induced by the Levi-Civita connection. The corresponding Dirac operator $D$ is the classical Dirac operator.

Example 3. Let $M$ be a $\operatorname{spin}^{c}$ manifold. Again, there exists the complex spinor bundle $S$ of rank $2^{[n / 2]}$. The connection $\nabla$ depends on the Levi-Civita connection and the choice of a connection on a certain $\mathrm{U}(1)$-bundle, the determinant bundle.

Example 4. Let $M$ be an almost complex manifold. Then $M$ is canonically $\operatorname{spin}^{c}$ and the spinor bundle of example 3 can be identified with the bundle of mixed $(0, p)$-forms, $S=\Lambda^{0, *}(T M \otimes \mathbb{C})$. The Dirac operator is given by $D=$ $\sqrt{2} \cdot\left(\bar{\partial}+\bar{\partial}^{*}\right)+h$ where $h$ is a zero order term which vanishes if $M$ is Kähler.

Example 5. Let $S$ be a Dirac bundle over $M$, and let $E$ be a Riemannian or Hermitian bundle over $M$ with metric connection. Then $S \otimes E$ canonically becomes a Dirac bundle and the corresponding Dirac operator is called twisted Dirac operator with coefficients in E.

Let us see what the theorem tells us when applied to the example of differential forms. First of all, we see that the bound in the theorem is optimal. Namely, let $F$ be a surface of higher genus, let $\omega_{1}$ be a nontrivial closed and coclosed 1 -form on $F$. In other words, $\omega_{1}$ is a solution of $(d+\delta) \omega_{1}=0$. The nodal set of $\omega_{1}$ consists of isolated points and it is nonempty since the Euler number of $F$ is nonzero. Let $T^{n-2}$ be a flat $(n-2)$-torus and let $\omega_{2}$ be a parallel $k$-form on $T^{n-2}$. Put $M=F \times T^{n-2}$ and denote the projections onto the factors by

$\pi_{1}: M \rightarrow F$ and $\pi_{2}: M \rightarrow T^{n-2}$. Then $\omega=\pi_{1}^{*} \omega_{1} \wedge \pi_{2}^{*} \omega_{2}$ is a closed and coclosed $(k+1)$-form on $M$. Its nodal set is a disjoint union of copies of $T^{n-2}$ and therefore has codimension 2 in $M$.

Corollary 1. Let $M$ be a compact connected Riemannian manifold without boundary. Let $\Delta=d \delta+\delta d$ be the Laplace-Beltrami operator acting on $k$-forms. 
Let $\omega \not \equiv 0$ be a harmonic $k$-form, i.e. $\Delta \omega=0$.

Then the nodal set of $\omega$ is a countably $(n-2)$-rectifiable set and thus has Hausdorff dimension $n-2$ at most.

If $n=2$, then the nodal set of $\omega$ is a discrete subset of $M$.

PROOF. Taking $L^{2}$-products and using the Green formula (3) yields

$$
\begin{aligned}
0 & =(\Delta \omega, \omega)_{L^{2}(M)} \\
& =\left((d+\delta)^{2} \omega, \omega\right)_{L^{2}(M)} \\
& =((d+\delta) \omega,(d+\delta) \omega)_{L^{2}(M)}
\end{aligned}
$$

We conclude

$$
(d+\delta) \omega \equiv 0 .
$$

Now the theorem tells us that the nodal set of $\omega$ is a countably $(n-2)$-rectifiable set.

It is remarkable that Corollary 1 fails if we drop the assumption that $M$ be compact. For example, $\omega=x_{1} d x_{1} \wedge \ldots \wedge d x_{k}$ is a harmonic $k$-form on $\mathbb{R}^{n}$ whose nodal set has codimension 1 . This means that despite the local nature of the statement of Corollary 1 it can not be proved by purely local methods.

One can extend Corollary 1 to complete noncompact manifolds by imposing suitable decay conditions on the form $\omega$. This will make the additional boundary term $\int_{B(R)}\langle\nu \cdot(d+\delta) \omega, \omega\rangle$ tend to zero as $R \rightarrow \infty$ where $B(R)$ denotes the distance ball of radius $R$ around some fixed point. Demanding $\omega$ to be in the Sobolev space $H^{\frac{1}{2}, 2}(M)$ should be sufficient.

Corollary 1 also fails if we replace harmonic forms by other eigenforms of the Laplace-Beltrami operator even if the manifold is compact. For example, $\omega=\sin \left(2 \pi m x_{1}\right) d x_{1} \wedge \ldots \wedge d x_{k}, m \in \mathbb{Z}$, is a $\Delta$-eigenform on the torus $T^{n}=\mathbb{R}^{n} / \mathbb{Z}^{n}$ whose nodal set has codimension 1 .

As another application of the main theorem we obtain a quick proof of the following theorem first proven 1989 by R. Hardt and L. Simon [18, Theorem 1.10].

Corollary 2. Let $M$ be an n-dimensional connected Riemannian manifold. Let $f$ be a nontrivial $\Delta$-eigenfunction on $M$, i.e.

$$
\Delta f=\lambda f
$$

for some $\lambda>0$.

Then the nodal set of $f$ is a disjoint union $N_{\text {reg }} \cup N_{\text {sing }}$ where $N_{\text {reg }}$ is a smooth hypersurface of $M$ and $N_{\text {sing }}$ is a countably $(n-2)$-rectifiable set and thus has Hausdorff dimension $n-2$ at most. 
ProOF. Put $N_{\text {reg }}=\{x \in M \mid f(x)=0, d f(x) \neq 0\}$ and $N_{\text {sing }}=\{x \in$ $M \mid f(x)=0, d f(x)=0\}$. Then the nodal set of $f$ is given by $N_{\text {reg }} \cup N_{\text {sing }}$. By the implicit function theorem $N_{\text {reg }}$ is a smooth hypersurface.

The other set $N_{\text {sing }}$ is the zero locus of the mixed differential form $\omega=\sqrt{\lambda} f+$ $d f$. Now $\omega$ is an eigenform for the generalized Dirac operator $D=d+\delta$,

$$
(d+\delta) \omega=\sqrt{\lambda} \omega .
$$

The main theorem says $N_{\text {sing }}$ is a countably $(n-2)$-rectifiable set.

Remark. If we knew that Corollary 2 is true for generalized Laplacians, then the main theorem could be derived from it, at least for eigensections $s$ of a Dirac operator, as follows.

Let $D s=\lambda s, \lambda \in \mathbb{R}$. The Weitzenböck formula (2) yields

$$
\nabla^{*} \nabla s+\left(\Re-\lambda^{2}\right) s=\left(D^{2}-\lambda^{2}\right) s=0 .
$$

Hence we could conclude that the nodal set of $s$ is of the form $N_{\text {reg }} \cup N_{\text {sing }}$ where $N_{\text {reg }}$ is a smooth hypersurface and $N_{\text {sing }}$ has at least codimension 2. It would remain to show $N_{\text {reg }}=\emptyset$.

Assume there is $x_{0} \in N_{\text {reg }}$. Choose a small ball $B$ around $x_{0}$ which is cut by $N_{\text {reg }}$ into two pieces $B_{1}$ and $B_{2}$. Define

$$
\tilde{s}(x)=\left\{\begin{array}{l}
s(x), \text { if } x \in B_{1}, \\
0, \text { if } x \in B_{2} .
\end{array}\right.
$$

Then $\tilde{s}$ is a continuous section of $S$ over $B$. Let $\phi$ be a smooth test section of $S$ with compact support contained in $B$. Let $\nu$ be the unit normal field of $N_{\text {reg }} \cap B$ pointing into $B_{2}$.

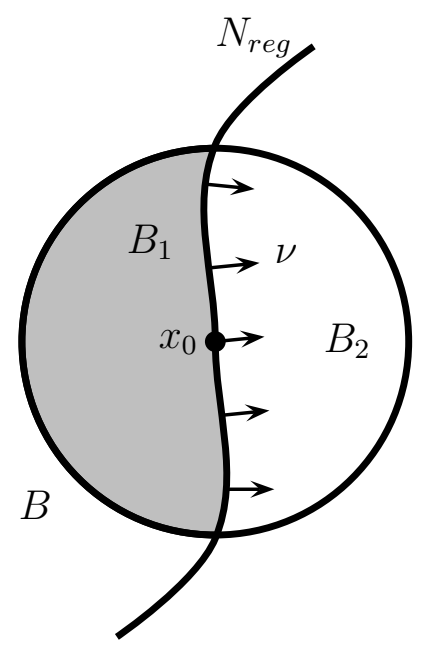

Fig. 1 
Then by (3)

$$
\begin{aligned}
(\tilde{s}, D \phi)_{L^{2}(B)} & =(s, D \phi)_{L^{2}\left(B_{1}\right)} \\
& =(D s, \phi)_{L^{2}\left(B_{1}\right)}+\int_{B \cap N_{r e g}}\langle s, \nu \cdot \phi\rangle \\
& =(\lambda s, \phi)_{L^{2}\left(B_{1}\right)}+0 \\
& =(\lambda \tilde{s}, \phi)_{L^{2}(B)} .
\end{aligned}
$$

Hence $D \tilde{s}=\lambda \tilde{s}$ holds in $B$ in the sense of distributions. By elliptic regularity theory $\tilde{s}$ is smooth. Since $\tilde{s}$ vanishes identically on $B_{2}$ we know from Aronszajn's unique continuation theorem (see next section) that $\tilde{s} \equiv 0$ on $B$. Hence $s \equiv 0$ on $B_{1}$. Applying Aronszajn's theorem once more we conclude $s \equiv 0$ on $M$.

Unfortunately, to our knowledge Corollary 2 is not established for generalized Laplacians. But, using the methods of the next section, it is easy to see that nodal sets for generalized Laplacians have Hausdorff dimension $n-1$ at most.

Note that the nodal set of a solution of a general linear elliptic system of second order can be very irregular. For example, it is not hard to show the following.

Let $A \subset \mathbb{R}^{n-1}$ be any closed subset. Then there is a linear elliptic differential operator of second order, $P$, acting on functions $u: \mathbb{R}^{n} \rightarrow \mathbb{R}^{2}$ and there is a solution $u$ of $P u=0$ such that $u^{-1}(0)=A \times\{0\} \subset \mathbb{R}^{n}$.

To conclude this section let us emphasize once more the two-dimensional case. We have the following generalization of the well-known uniqueness theorem for holomorphic functions.

Corollary 3. Let $M$ be a two-dimensional connected Riemannian manifold. If the zero set of a solution of a generalized Dirac equation on $M$ has a cumulation point, then this solution must vanish identically.

This corollary has been proven for many special cases. The Dirac equation on a surface can be written as a generalized Cauchy-Riemann equation. If for example the real dimension of the Dirac bundle is 2, then the theory of "generalized analytic functions" applies, see [25], also compare [9].

\section{Proof of the Main Theorem}

This section is devoted to the proof of the main theorem. There are two important ingredients to the proof which we state first. We have

Aronszajn's Unique Continuation Theorem. Let $M$ be a connected Riemannian manifold. Let $L$ be an operator of the form $L=\nabla^{*} \nabla+L_{1}+L_{0}$ 
acting on sections of a vector bundle $S$ over $M$ where $L_{1}$ and $L_{0}$ are differential operators of first and zero ${ }^{\text {th }}$ order respectively. Let $s$ be a solution of

$$
L s=0 .
$$

If $s$ vanishes at some point of infinite order, i.e. if all derivatives vanish at that point, then $s \equiv 0$.

For a proof see [2, Theorem on p. 235 and Remark 3 on p. 248]. The other essential ingredient is a version of Weierstrass' preparation theorem for differentiable functions [22, Chapter V], [6, 6.3].

Malgrange's Preparation Theorem (Special case). Let $U \subset \mathbb{R}^{n}$ be an open neighborhood of 0 , let $f: U \rightarrow \mathbb{R}$ be a $C^{\infty}$-function vanishing of $k^{\text {th }}$ order at 0 but not of $(k+1)^{\text {st }}$ order, $k \in \mathbb{N}$. Then, after possibly shrinking $U$ to a smaller neighborhood and applying a linear coordinate transformation to $\mathbb{R}^{n}$, there exist $C^{\infty}$-functions $v: U \rightarrow \mathbb{R}$ and $u_{j}: U \cap\left(\{0\} \times \mathbb{R}^{n-1}\right) \rightarrow \mathbb{R}$ such that

$$
f(x)=v(x) \cdot\left(x_{1}^{k}+\sum_{j=0}^{k-1} u_{j}\left(x^{\prime}\right) x_{1}^{j}\right)
$$

for all $x=\left(x_{1}, x^{\prime}\right) \in U$ where $v(x) \neq 0$ for all $x \in U$ and $u_{j}$ vanishes of order $k-j$ at $0 \in \mathbb{R}^{n-1}$.

Remark. By Taylor's theorem we can write $f=\hat{f}+\psi$ where $\hat{f}$ is a homogeneous polynomial of degree $k$ and $\psi$ vanishes of order $k+1$ at 0 . The linear coordinate transformation in the Preparation Theorem must be such that a vector $w \in \mathbb{R}^{n}$ for which $\hat{f}(w) \neq 0$ is transformed into $(1,0, \ldots, 0)$.

PRoOf OF MAIN THEOREM. To prove the main theorem let $s$ be a section of the Dirac bundle satisfying

$$
(D+h) s=0 .
$$

We assume that the Dirac bundle is real, in the complex case we simply forget the complex structure.

Let $p \in M$ be a point of its nodal set, i.e. $s(p)=0$. Applying $D$ to (4) and using the Weitzenböck formula we get (2)

$$
\left(\nabla^{*} \nabla+D \circ h+\Re\right) s=0 .
$$

By Aronszajn's unique continuation theorem $s$ cannot vanish of infinite order at $p$. Say $s$ vanishes at $p$ of order $k$ but not of order $k+1$.

We choose normal coordinates around $p$ and trivialize the Dirac bundle. Then $s$ corresponds to a vector valued function $\left(s_{1}, \ldots, s_{r}\right)$ defined in a neighborhood 
of 0 . Here $r$ is the (real) rank of the Dirac bundle $S$. All component functions vanish of order $k$ at $p$ and at least one of them does not vanish of order $k+$ 1. Other components could a-priori vanish of higher order but by choosing the trivialization appropriately we can assume that this is not the case. To see this, note that trivializing the bundle amounts to exhibiting linearly independent linear functionals on the bundle. Pick one linear functional $l_{1}$ such that $s_{1}=l_{1} \circ s$ does not vanish of order $k+1$ at 0 . Choose the other $r-1$ functionals linearly independent but close to $l_{1}$.

Moreover, if the Dirac bundle is trivialized in this way, then there is a direction in which the $k^{\text {th }}$ derivative of all components $s_{1}, \ldots, s_{r}$ does not vanish. Hence we can use Malgrange's preparation theorem with the same linear coordinate transformation (the same $x_{1}$-direction) for all the components. Therefore we can write

$$
s_{m}(x)=v_{m}(x) \cdot\left(x_{1}^{k}+\sum_{j=0}^{k-1} u_{m, j}\left(x^{\prime}\right) x_{1}^{j}\right)
$$

where $v_{m}$ are nonvanishing and $u_{m, j}$ vanish of order $k-j$ at $0, m=1, \ldots, r$.

We see already that the nodal set of $s$ near $p$ is countably $(n-1)$-rectifiable. Namely, for any $m$ the nodal set is contained in the set

$$
N_{m}=\left\{x=\left(x_{1}, x^{\prime}\right) \mid x_{1}^{k}+\sum_{j=0}^{k-1} u_{m, j}\left(x^{\prime}\right) x_{1}^{j}=0\right\} .
$$

That this set $N_{m}$ is countably $(n-1)$-rectifiable follows easily from the following

Fact. $\mathbb{R}^{k}$ can be written as a disjoint union of countably many bounded subsets $A_{\nu}$ such that the number of pairwise distinct real roots of the polynomial $P_{u}(t)=t^{k}+\sum_{j=0}^{k-1} u_{j} t^{j}$ is constant for $u=\left(u_{0}, \ldots, u_{k-1}\right) \in A_{\nu}$ and the real roots (ordered my magnitude) are Lipschitz-functions on $A_{\nu}$.

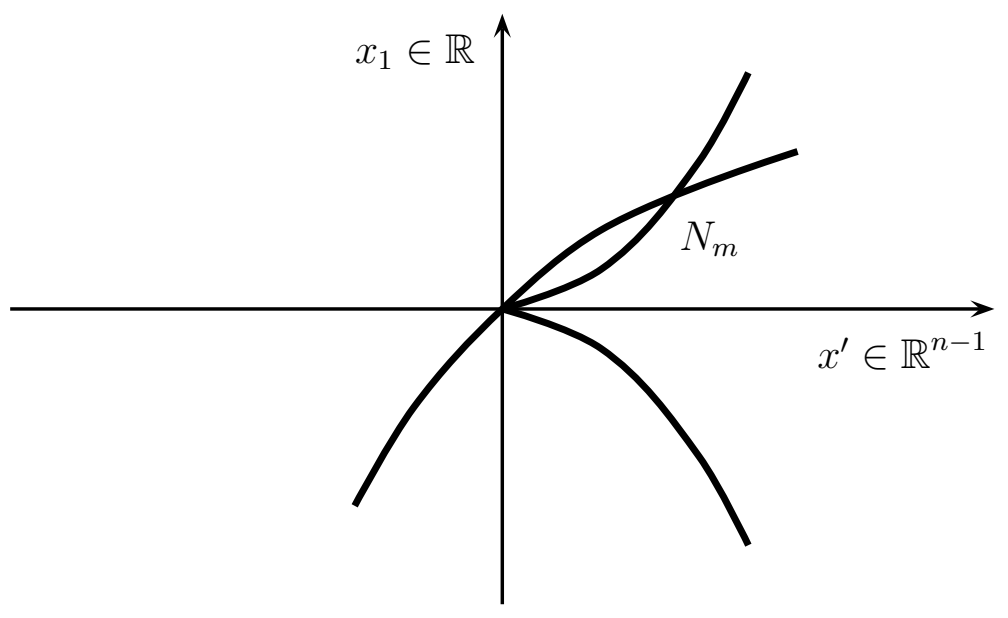

Fig. 2 
Recall that two polynomials $F=\sum_{j=0}^{k} a_{j} t^{j}$ and $G=\sum_{j=0}^{k} b_{j} t^{j}$ have a common root if and only if the resultant $R_{F, G}$ vanishes. The resultant is a weighted homogeneous polynomial of degree $k^{2}$ in the coefficients $a_{j}$ and $b_{j}$ where $a_{j}$ and $b_{j}$ have weight $k-j$, see [0, Section 4].

More generally, $r$ polynomials $P_{m}=\sum_{j=0}^{k} u_{m, j} t^{j}, m=1, \ldots, r$ have a common root if and only if any two linear combinations $F=\sum_{m=1}^{r} \alpha_{m} P_{m}$ and $G=\sum_{m=1}^{r} \beta_{m} P_{m}$ have vanishing resultant $R_{F, G}$.

We will show that there are two linear combinations $F=\sum_{m=1}^{r} \alpha_{m} v_{m}(0) P_{m}$ and $G=\sum_{m=1}^{r} \beta_{m} v_{m}(0) P_{m}$ of our polynomials $P_{m}=t^{k}+\sum_{j=0}^{k-1} u_{m, j}\left(x^{\prime}\right) t^{j}$ such that the resultant $R_{F, G}$ regarded as a function in $x^{\prime}$ does not vanish of infinite order at $x^{\prime}=0$. Then, by applying once more Malgrange's preparation theorem and the fact on the real roots of polynomials mentioned above, we see that the zero locus of $R_{F, G}$ is a countably $(n-2)$-rectifiable subset of $\mathbb{R}^{n-1}$ and that $N=\cap_{m=1}^{r} N_{m}$ is contained in a countably $(n-2)$-rectifiable subset of $\mathbb{R}^{n}$. Moreover, if $n=2$, we see that $x=0$ is an isolated zero of $s$ and the theorem is proved.

To find two such linear combinations $F$ and $G$ we look at the Taylor expansion $v_{m}\left(x^{\prime}\right)=v_{m}(0)+$ first order terms, $u_{m, j}\left(x^{\prime}\right)=\hat{u}_{m, j}\left(x^{\prime}\right)+$ higher order terms. Here $\hat{u}_{m, j}\left(x^{\prime}\right)$ is a homogeneous polynomial of degree $k-j$. The Taylor expansion of $s_{m}$ is then given by

$$
s_{m}(x)=v_{m}(0) \cdot\left(x_{1}^{k}+\sum_{j=0}^{k-1} \hat{u}_{m, j}\left(x^{\prime}\right) x_{1}^{j}\right)+\text { higher order terms. }
$$

Looking at the lowest order term of the left hand side of equation (iㅣ) we get

$$
\hat{D} w=0
$$

where $w(x)=\left(w_{1}(x), \ldots, w_{r}(x)\right), w_{m}(x)=v_{m}(0) \cdot\left(x_{1}^{k}+\sum_{j=0}^{k-1} \hat{u}_{m, j}\left(x^{\prime}\right) x_{1}^{j}\right)$ and $\hat{D}=\sum_{i=1}^{n} \gamma_{i} \partial / \partial x_{i}$ is the Dirac operator on $T_{p} M=\mathbb{R}^{n}$. Here $\gamma_{i}$ are generalized Pauli matrices satisfying the relations $\gamma_{i} \gamma_{j}+\gamma_{j} \gamma_{i}=-2 \delta_{i j}$.

Putting $y_{j}\left(x^{\prime}\right)=\left(v_{1}(0) \hat{u}_{1, j}\left(x^{\prime}\right), \ldots, v_{r}(0) \hat{u}_{r, j}\left(x^{\prime}\right)\right)$ for $j=0, \ldots, k-1$ and $y_{k}\left(x^{\prime}\right)=\left(v_{1}(0), \ldots, v_{r}(0)\right)$ we have

$$
w(x)=\sum_{j=0}^{k} y_{j}\left(x^{\prime}\right) x_{1}^{j} .
$$

Write $D_{1}=\sum_{j=2}^{n} \gamma_{1} \gamma_{j} \partial / \partial x_{j}$. Then $D_{1}$ is a generalized Dirac operator on $\mathbb{R}^{n-1}$. Plugging (7) into (6) and comparing coefficients of $x_{1}^{j}$ yields

$$
\begin{aligned}
& D_{1} y_{j}=(j+1) \cdot y_{j+1}, \quad j=0, \ldots, k-1 \\
& D_{1} y_{k}=0 .
\end{aligned}
$$

Hence

$$
y_{j}=\frac{1}{j !} D_{1}^{j} y_{0}, \quad j=0, \ldots, k .
$$


Given two linear combinations $\hat{F}=\sum_{m=1}^{r} \alpha_{m} w_{m}$ and $\hat{G}=\sum_{m=1}^{r} \beta_{m} w_{m}$ the resultant $R_{\hat{F}, \hat{G}}$ is precisely the lowest order term of the resultant of the corresponding linear combinations of the $P_{m}$ 's, $F=\sum_{m=1}^{r} \alpha_{m} v_{m}(0) P_{m}, G=$ $\sum_{m=1}^{r} \beta_{m} v_{m}(0) P_{m}$,

$$
R_{F, G}\left(x^{\prime}\right)=R_{\hat{F}, \hat{G}}\left(x^{\prime}\right)+\text { higher order terms. }
$$

To show that $R_{F, G}$ does not vanish of infinite order at $x^{\prime}=0$ it is thus sufficient to show that $R_{\hat{F}, \hat{G}}$ does not vanish identically. Therefore the theorem is proved if we can choose the $\alpha_{m}$ and $\beta_{m}$ in such a way that $R_{\hat{F}, \hat{G}}$ does not vanish identically.

Assume the resultant $R_{\hat{F}, \hat{G}}$ vanishes for all linear combinations $\hat{F}$ and $\hat{G}$. Then for any $x^{\prime} \in \mathbb{R}^{n-1}$ there exists a common root $\xi\left(x^{\prime}\right)$ of the polynomials $w_{1}, \ldots, w_{r}$. By (7) and (8) we have

$$
\sum_{j=0}^{k} \frac{1}{j !} D_{1}^{j} y_{0}\left(x^{\prime}\right) \xi\left(x^{\prime}\right)^{j}=0 .
$$

There is a nonempty open subset of $\mathbb{R}^{n-1}$ on which $\xi$ can be chosen such that it depends smoothly on $x^{\prime}$. On this subset we apply $D_{1}$ to (10) and use (1) to obtain

$$
\sum_{j=0}^{k-1} \frac{\xi\left(x^{\prime}\right)^{j}}{j !} \cdot(1+\nabla \xi) \cdot D_{1}^{j+1} y_{0}\left(x^{\prime}\right)=0 .
$$

If $v \in V$ is a vector in a finite dimensional Euclidean vector space $V$, then the element $1+v$ is invertible in the Clifford algebra $C l(V)$ with inverse $\frac{1-v}{1+|v|^{2}}$. Thus (11) gives

$$
\sum_{j=0}^{k-1} \frac{\xi\left(x^{\prime}\right)^{j}}{j !} D_{1}^{j+1} y_{0}\left(x^{\prime}\right)=0 .
$$

Repeating this argument inductively we eventually get

$$
D_{1}^{k} y_{0}=0
$$

But this means $y_{k}\left(x^{\prime}\right)=\left(v_{1}(0), \ldots, v_{r}(0)\right)=0$, a contradiction.

\section{References}

[1] J. H. Albert, Nodal and Critical Sets for Eigenfunctions of Elliptic Operators, Proc. Symp. Pure Math. 23, 71-78 (1973)

[2] N. Aronszajn, A Unique Continuation Theorem for Solutions of Elliptic Partial Differential Equations or Inequalities of Second Order, J. Math. Pures Appl. 36, 235-249 (1957) 
[3] P. Bérard, D. Meyer, Inégalités isopérimétriques et applications, Ann. Sci. Éc. Norm. Sup. 15, 513-542 (1982)

[4] N. Berline, E. Getzler, M. Vergne, Heat Kernels and Dirac Operators, Springer-Verlag, Berlin Heidelberg 1992

[5] E. Brieskorn, H. Knörrer, Plane Algebraic Curves, Birkhäuser Verlag, Basel 1986

[6] T. Bröcker, L. Lander, Differentiable Germs and Catastrophes, Cambridge Univ. Press, Cambridge 1975

[7] J. Brüning, Über Knoten von Eigenfunktionen des Laplace-BeltramiOperators, Math. Z. 158, 15-21 (1978)

[8] L. Caffarelli, A. Friedman, Partial Regularity of the Zero Set of Linear and Superlinear Elliptic Equations, J. Diff. Eq. 60, 420-439 (1985)

[9] T. Carleman, Sur les systèmes linéaires aux dérivées partielles du premier ordre à deux variables, C. R. Acad. Sci. Paris 197, 471-474 (1933)

[10] S. Chanillo, B. Muckenhoupt, Nodal Geometry on Riemannian Manifolds, J. Diff. Geom. 34, 85-91 (1991)

[11] J. Chen, The Local Structure of Nodal Set of Solutions of Schroedinger Equation on Riemannian 3-manifolds, Manuscr. Math. 85, 255-263 (1994)

[12] S.-Y. Cheng, Eigenfunctions and Nodal Sets, Comment. Math. Helv. 51, 43-55 (1976)

[13] R.-T. Dong, Nodal Sets of Eigenfunctions on Riemann Surfaces, J. Diff. Geom. 36, 493-506 (1992)

[14] H. Donnelly, Nodal Sets for Sums of Eigenfunctions on Riemannian Manifolds, Proc. Am. Math. Soc. 121, $967-973$ (1994)

[15] H. Donnelly, C. Fefferman, Nodal Sets of Eigenfunctions on Riemannian Manifolds, Invent. Math. 93, 161-183 (1988)

[16] H. Donnelly, C. Fefferman, Nodal Sets of Eigenfunctions: Riemannian Manifolds with Boundary, in: Analysis, et cetera, Res. Pap. in Honor of J. Moser's 60 ${ }^{\text {th }}$ Birthd. 251-262 (1990)

[17] H. Donnelly, C. Fefferman, Nodal Sets for Eigenfunctions of the Laplacian on Surfaces, J. Am. Math. Soc. 3, 333-353 (1990)

[18] R. Hardt, L. Simon, Nodal Sets for Solutions of Elliptic Equations, J. Diff. Geom. 30, 505-522 (1989) 
[19] D. Kotschick, The Seiberg-Witten Invariants of Symplectic Four-Manifolds, Astérisque (to appear)

[20] H. B. Lawson, M.-L. Michelsohn, Spin Geometry, Princeton Univ. Press, Princeton 1989

[21] G. Lu, Covering Lemmas and an Application to Nodal Geometry on Riemannian Manifolds, Proc. Am. Math. Soc. 117, 971-978 (1993)

[22] B. Malgrange, Ideals of Differentiable Functions, Oxford Univ. Press, Oxford 1966

[23] C.H. Taubes, The Seiberg-Witten and Gromov Invariants, Math. Res. Letters 2, 221-238 (1995)

[24] C.H. Taubes, $S W \Rightarrow G r$, From the Seiberg-Witten Equations to Pseudoholomorphic Curves, J. Am. Math. Soc. (to appear)

[25] I.N. Vekua, Generalized Analytic Functions, Pergamon Press, Oxford 1962

Mathematisches Institut

Universität Freiburg

Eckerstr. 1

79104 Freiburg

Germany

e-mail: baer@mathematik. uni-freiburg.de 